\title{
Electromagnetic polarizabilities and the excited states of the nucleon
}

\author{
Martin Schumacher \\ mschuma3@gwdg.de \\ Zweites Physikalisches Institut der Universität Göttingen, Friedrich-Hund-Platz 1 \\ D-37077 Göttingen, Germany
}

\begin{abstract}
The electromagnetic polarizabilities of the nucleon are shown to be essentially composed of the nonresonant $\alpha_{p}\left(E_{0+}\right)=+3.2, \alpha_{n}\left(E_{0+}\right)=+4.1$, the $t$-channel $\alpha_{p, n}^{t}=-\beta_{p, n}^{t}=+7.6$ and the resonant $\beta_{p, n}\left(P_{33}(1232)\right)=+8.3$ contributions (in units of $10^{-4} \mathrm{fm}^{3}$ ). The remaining deviations from the experimental data $\Delta \alpha_{p}=1.2 \pm 0.6, \Delta \beta_{p}=1.2 \mp 0.6, \Delta \alpha_{n}=0.8 \pm 1.7$ and $\Delta \beta_{n}=2.0 \mp 1.8$ are contributed by a larger number of resonant and nonresonant processes with cancellations between the contributions. This result confirms that dominant contributions to the electric and magnetic polarizabilities may be represented in terms of twophoton coupling to the $\sigma$ meson having the predicted mass $m_{\sigma}=666 \mathrm{MeV}$ and two-photon width $\Gamma_{\gamma \gamma}=2.6 \mathrm{keV}$.
\end{abstract}

PACS. 11.55.Fv Dispersion relations - 11.55.Hx Sum rules - 13.60.Fz Elastic and Compton scattering - 14.70.Bh Photons

\section{Introduction}

Recently, it has been shown [1] that the $t$-channel components $\alpha^{t}$ and $\beta^{t}$ of the electric $(\alpha)$ and magnetic $(\beta)$ polarizabilities of the nucleon can be understood as a property of the constituent quarks. The constituent quarks couple to $\pi$ and $\sigma$ fields and, mediated by these fields, they couple to two photons. The coupling of two photons with perpendicular linear polarization to the $\pi^{0}$ meson provides the main contribution, $\gamma_{\pi}^{t}$, to the backward-angle spin-polarizability $\gamma_{\pi}$. Similarly, the coupling of two photons with parallel linear polarization provides the main contribution, $(\alpha-\beta)^{t}$, to the difference $(\alpha-\beta)$ of the electric and the magnetic polarizabilities. The quantitative prediction $(\alpha-\beta)_{p, n}^{t}=15.2$ (in units of $10^{-4} \mathrm{fm}^{3}$ ) makes use of the fact that the mass of the particle of the $\sigma$ field is predicted by the quark-level Nambu-Jona-Lasinio (NJL) model to be $m_{\sigma}=666 \mathrm{MeV}$ and its two-photon width to be $\Gamma_{\gamma \gamma}=2.6 \mathrm{keV}$ [1].

The foregoing paragraph describes the résumé of a long and partial controversial history of research. The scalar-isoscalar $t$-channel was introduced [2] in analogy to the pseudoscalar $t$-channel [3]. But differing from the $\pi^{0}$-pole contribution [3] to the scattering amplitude, the meaning and importance of the scalar-isoscalar $t$-channel [2] was less well known, mainly because the $\sigma$ meson was not considered as a normal particle. One important step forward was the formulation of the BEFT sum rule [4], relating the $s$-channel part of the difference of the electric and magnetic polarizabilities, $(\alpha-\beta)^{s}$, to the multipole content of the total photoabsorption cross section using a fixed- $\theta$ dispersion relation at $\theta=\pi$, and by relating the $t$-channel part $(\alpha-\beta)^{t}$ to a dispersion relation for $t$ with the imaginary part of the amplitude taken from the reactions $\gamma \gamma \rightarrow \pi \pi$ and $N \bar{N} \rightarrow \pi \pi$ via a unitarity relation. Furthermore, the scalar-isoscalar phase $\delta_{0}^{0}(t)$ was taken from the reaction $\pi \pi \rightarrow \pi \pi$. One of the first evaluations of the BEFT sum-rule showed that for pointlike uncorrelated pions the large value of $(\alpha-\beta)^{t}=+17.51$ is obtained [5] which in other calculations has been reduced by very different factors (see [6] for an overview) when the $\pi \pi$ correlation and the pion internal structure is taken into account. The largest reduction amounting to a factor of 2 has been obtained in the latest of this early series of 
calculations [7]. This unsatisfactory situation has recently been clarified by showing $[1,8]$ that the arithmetic average of the most recent calculations of Drechsel et al. [9] and Levchuk et al. (see $[6]),(\alpha-\beta)_{p, n}^{t}=15.3 \pm 1.3$, leads to a very good agreement with the experimental result and with a parameter-free calculation based on the quark-level NJL model or dynamical linear $\sigma$ model $(\mathrm{L} \sigma \mathrm{M})[1,10,11]$, leading to $(\alpha-\beta)_{p, n}^{t}=15.2$.

After the size and the dynamics of the $t$-channel contribution to the electromagnetic polarizabilities has been well understood, it appears of interest to get a similar understanding for the $s$-channel contribution. Especially, the question has to be answered what the individual contributions of the resonant excited states of the nucleon to the electric and magnetic polarizabilities are and how the contributions of the "pion cloud" to the electric and magnetic polarizabilities may be specified. To the author's knowledge such an investigation has not been carried out before.

\section{Electromagnetic polarizabilities obtained from the forward- angle sum-rule for $(\alpha+\beta)$ and the backward-angle sum-rule for $(\alpha-\beta)$}

The appropriate tool for the present investigation is to simultaneously apply the forward-angle sum-rule for $(\alpha+\beta)$ and the backward-angle sum-rule for $(\alpha-\beta)$. This leads to the following relations

$$
\begin{aligned}
& \alpha=\alpha^{s}+\alpha^{t}, \\
& \alpha^{s}=\frac{1}{2 \pi^{2}} \int_{\omega_{0}}^{\infty}[A(\omega) \sigma(\omega, E 1, M 2, \cdots)+B(\omega) \sigma(\omega, M 1, E 2, \cdots)] \frac{d \omega}{\omega^{2}}, \\
& \alpha^{t}=\frac{5 \alpha_{e} g_{\pi N N}}{12 \pi^{2} m_{\sigma}^{2} f_{\pi}}=7.6,
\end{aligned}
$$

and

$$
\begin{aligned}
& \beta=\beta^{s}+\beta^{t} \\
& \beta^{s}=\frac{1}{2 \pi^{2}} \int_{\omega_{0}}^{\infty}[A(\omega) \sigma(\omega, M 1, E 2, \cdots)+B(\omega) \sigma(\omega, E 1, M 2, \cdots)] \frac{d \omega}{\omega^{2}}, \\
& \beta^{t}=-\frac{5 \alpha_{e} g_{\pi N N}}{12 \pi^{2} m_{\sigma}^{2} f_{\pi}}=-7.6 \\
& \omega_{0}=m_{\pi}+\frac{m_{\pi}^{2}}{2 m} \\
& A(\omega)=\frac{1}{2}\left(1+\sqrt{1+\frac{2 \omega}{m}}\right) \\
& B(\omega)=\frac{1}{2}\left(1-\sqrt{1+\frac{2 \omega}{m}}\right) .
\end{aligned}
$$

In (11) - (9) $\omega$ is the photon energy in the lab-system, $m_{\pi}$ the pion mass and $m$ the nucleon mass. The quantities $\alpha^{s}, \beta^{s}$ are the $s$-channel electric and magnetic polarizabilities, and $\alpha^{t}, \beta^{t}$ the $t$-channel electric and magnetic polarizabilities, respectively. The multipole content of the 
photoabsorption cross section enters through

$$
\begin{aligned}
\sigma(\omega, E 1, M 2, \cdots) & =\sigma(\omega, E 1)+\sigma(\omega, M 2)+\cdots \\
\sigma(\omega, M 1, E 2, \cdots) & =\sigma(\omega, M 1)+\sigma(\omega, E 2)+\cdots
\end{aligned}
$$

i.e. through the sums of cross sections with change and without change of parity during the electromagnetic transition, respectively 1 . The multipoles belonging to parity-change are favored for the electric polarizability $\alpha^{s}$ whereas the multipoles belonging to parity-nonchange are favored for the magnetic polarizability $\beta^{s}$. The coefficients $A(\omega)$ and $B(\omega)$ in Eqs. (2), (15), (8) and (9) multiplying the cross sections of the parity-favored and parity-nonfavored multipoles, respectively, are $A \sim+1.07$ and $B \sim-0.07$ at the pion photoproduction threshold. They increase with photon energy, as expected for relativistic correction factors. Using $A(\omega)$ and $B(\omega)$ it is easy to prove that $(\alpha+\beta) \equiv(\alpha+\beta)^{s}$ is given by the Baldin or Baldin-Lapidus (BL) [14] sum rule, whereas $(\alpha-\beta)^{s}$ is given by the $s$-channel part of the BEFT [4] sum rule.

For the $t$-channel parts, $\alpha^{t}$ and $\beta^{t}$, we use the predictions obtained from the $\sigma$-meson pole representation 2 with properties as predicted by the quark-level Nambu-Jona-Lasinio model [1, 8]. The quantities entering into this prediction are $\alpha_{e}=e^{2} / 4 \pi=1 / 137.04$, the pion-nucleon coupling constant, $g_{\pi N N}=13.169 \pm 0.057$, the pion decay constant, $f_{\pi}=(92.42 \pm 0.26) \mathrm{MeV}$, and the $\sigma$-meson mass, $m_{\sigma}=666.0 \mathrm{MeV}[1,10,11]$. For convenience we summarize the arguments leading to the relations (3) and (6) . The flavor wave-functions of the $\pi^{0}$ and the $\sigma$ meson are given by

$$
\left|\pi^{0}\right\rangle=\frac{1}{\sqrt{2}}(-u \bar{u}+d \bar{d}), \quad|\sigma\rangle=\frac{1}{\sqrt{2}}(u \bar{u}+d \bar{d}) .
$$

This leads to the decay matrix elements

$$
M(\sigma \rightarrow \gamma \gamma)=-\frac{5}{3} M\left(\pi^{0} \rightarrow \gamma \gamma\right)=\frac{5}{3} \frac{\alpha_{e}}{\pi f_{\pi}}
$$

Using the NJL model or the dynamical $\mathrm{L} \sigma \mathrm{M}$ with dimensional regularization we arrive at $[1,11]$

$$
m_{\sigma}^{\mathrm{cl}}=\frac{4 \pi f_{\pi}^{\mathrm{cl}}}{\sqrt{N_{c}}}
$$

where $m_{\sigma}^{\mathrm{cl}}$ and $f_{\pi}^{\mathrm{cl}}=89.8 \mathrm{MeV}$ are the $\sigma$ meson mass and the $\pi$ decay constant in the chiral limit (cl) and $N_{c}=3$ the number of colors. Then the mass of the $\sigma$ meson is given by

$$
m_{\sigma}=\sqrt{\left(m_{\sigma}^{\mathrm{cl}}\right)^{2}+m_{\pi}^{2}}=666 \mathrm{MeV}
$$

Inserting this into

$$
(\alpha-\beta)^{t}=\frac{g_{\sigma N N} M(\sigma \rightarrow \gamma \gamma)}{2 \pi m_{\sigma}^{2}}
$$

and using $f_{\sigma N N}=f_{\pi N N}$ and $(\alpha+\beta)^{t}=0$ we arrive at (3) and (마).

\footnotetext{
${ }^{1}$ It should be noted that this separation into cross sections for separate multipoles is possible in the presently used fixed- $\theta$ dispersion theory applied at $\theta=0$ and $\theta=\pi$, whereas in the corresponding formulas based on fixed- $t$ dispersion theory [12] terms containing mixed products of CGLN [13] amplitudes occur (for a discussion see [6]).

${ }^{2}$ This $\sigma$-meson pole in the complex $t$-plane of the Compton scattering amplitude $A(s, t)$ is not the same, but has relations with the $\sigma$-meson pole introduced to parameterize the $\pi \pi$ scattering amplitude. These relations have been discussed in detail in $[1,6]$.
} 


\section{Components of electromagnetic polarizabilities from analyses of total photoabsorption and meson photoproduction data}

In the following we use different photoabsorption data to get information on partial contributions to $\alpha^{s}$ and $\beta^{s}$. Analyses of total photoabsorption cross sections have been carried out in [15]. These analyses give a very good overview over the resonant and nonresonant contributions to the electromagnetic polarizabilities. Further information is taken from the PDG2006 [16], the GWSES [17] and the Mainz $[18,19]$ analyses of meson photoproduction data.

\subsection{Components of electromagnetic polarizabilities from analysis of the total photoabsorption cross-section of the proton}

In the following we wish to study the contributions of nucleon resonances and nonresonant excited states to the $s$-channel electromagnetic polarizabilities. Only the resonances $P_{33}(1232)$, $P_{11}(1440), D_{13}(1520), S_{11}(1535)$ and $F_{15}(1680)$ have to be taken into account. The contributions of the resonances $S_{11}(1650), D_{15}(1675)$ and higher lying resonances are negligible. For this analysis we use the Walker $[15,20]$ parameterization of nucleon resonances

$$
\begin{aligned}
& I=I_{r}\left(\frac{k_{r}}{k}\right)^{2} \frac{W_{r}^{2} \Gamma \Gamma_{\gamma}}{\left(W^{2}-W_{r}^{2}\right)^{2}+W_{r}^{2} \Gamma^{2}}, \\
& \Gamma=\Gamma_{r}\left(\frac{q}{q_{r}}\right)^{2 l+1}\left(\frac{q_{r}^{2}+X^{2}}{q^{2}+X^{2}}\right)^{l}, \\
& \Gamma_{\gamma}=\Gamma_{r}\left(\frac{k}{k_{r}}\right)^{2 j_{\gamma}}\left(\frac{k_{r}^{2}+X^{2}}{k^{2}+X^{2}}\right)^{j_{\gamma}} .
\end{aligned}
$$

$s=2 \omega m+m^{2}, \quad \omega=$ photon energy in the lab system,

$W^{2}=s$

$k=|\mathbf{k}|=\frac{s-m^{2}}{2 \sqrt{s}}, \quad|\mathbf{k}|=$ photon momentum in the c.m. system,

$q=|\mathbf{q}|=\sqrt{E_{\pi}^{2}-m_{\pi}^{2}} ; E_{\pi}=\frac{s-m^{2}+m_{\pi}^{2}}{2 \sqrt{s}},|\mathbf{q}|=\pi$ momentum in the c.m. system,

$j_{\gamma}$, multipole angular momentum of the photon,

$l$, single $\pi$ angular momentum.

The damping constants $X$ are $X=160 \mathrm{MeV}$ for the $P_{33}(1232)$ resonance and $X=350 \mathrm{MeV}$ else.

For the proton, parameters are given in [15] for the relevant resonant states, leading to the results given in lines $3-5$ of Table 1 . The sum $\alpha_{p}+\beta_{p}$ of nonresonant contributions in line 7 of Table 1 is in agreement with the corresponding number calculated from the nonresonant cross section given in [15] if the nonresonant cross section data are extrapolated to about $3.5 \mathrm{GeV}$. This shows that with the predicted $t$-channel contributions given in line 6 there is consistency between the experimental electromagnetic polarizabilities and the predictions.

\subsection{Components of electromagnetic polarizabilities from analyses of meson photoproduction for the proton and the neutron}

From isospin considerations it has been derived [21] that the amplitudes for meson photoproduction are composed of $A^{(1 / 2)}$ and $A^{(3 / 2)}$, referring to final states of definite isospin ( $\frac{1}{2}$ or $\left.\frac{3}{2}\right)$. 
Table 1: Partial contributions to the electromagnetic polarizabilities based on the analysis of the total photoabsorption cross section [15]. The $t$-channel parts in line 6 are the predictions based on the $\sigma$-meson pole representation (see section 2). Line 7 contains the differences between the numbers in line 2 and the sums of numbers given in lines $3-6$. The experimental data are normalized to $(\alpha+\beta)_{p}=13.9 \pm 0.3$ (see $[6]$ ).

\begin{tabular}{lll|ll|}
\hline 1 & & $\alpha_{p}$ & $\beta_{p}$ \\
\hline 2 & experiment & $12.0 \pm 0.6$ & $1.9 \mp 0.6$ \\
\hline 3 & $P_{33}(1232)$ & $M 1, E 2$ & -1.1 & +8.3 \\
4 & $P_{11}(1440)$ & $M 1$ & -0.1 & +0.3 \\
5 & $D_{13}(1520)$ & $E 1, M 2$ & +1.2 & -0.3 \\
6 & $S_{11}(1535)$ & $E 1$ & +0.1 & -0.0 \\
5 & $F_{15}(1680)$ & $E 2, M 3$ & -0.1 & +0.4 \\
6 & $t$-channel & +7.6 & -7.6 \\
7 & nonresonant & +4.4 & +0.8 \\
\hline
\end{tabular}

Furthermore, there is an amplitude $A^{(0)}$ which may be related to "recoil" effects [21]. This latter amplitude makes a contribution to $I=1 / 2$ only. Therefore, the amplitudes

$$
{ }_{p} A^{(1 / 2)}=A^{(0)}+\frac{1}{3} A^{(1 / 2)}, \quad{ }_{n} A^{(1 / 2)}=A^{(0)}-\frac{1}{3} A^{(1 / 2)}
$$

may be introduced. Furthermore, with

$$
A^{(+)}=\frac{1}{3}\left(A^{(1 / 2)}+2 A^{(3 / 2)}\right), \quad A^{(-)}=\frac{1}{3}\left(A^{(1 / 2)}-A^{(3 / 2)}\right),
$$

the physical amplitudes may be expressed by the isospin combinations (see e.g. [19,22])

$$
\begin{aligned}
& A\left(\gamma p \rightarrow n \pi^{+}\right)=\sqrt{2}\left(A^{(-)}+A^{(0)}\right)=\sqrt{2}\left({ }_{p} A^{(1 / 2)}-\frac{1}{3} A^{(3 / 2)}\right), \\
& A\left(\gamma p \rightarrow p \pi^{0}\right)=A^{(+)}+A^{(0)}={ }_{p} A^{(1 / 2)}+\frac{2}{3} A^{(3 / 2)}, \\
& A\left(\gamma n \rightarrow p \pi^{-}\right)=-\sqrt{2}\left(A^{(-)}-A^{(0)}\right)=\sqrt{2}\left({ }_{n} A^{(1 / 2)}+\frac{1}{3} A^{(3 / 2)}\right), \\
& A\left(\gamma n \rightarrow n \pi^{0}\right)=A^{(+)}-A^{(0)}=-{ }_{n} A^{(1 / 2)}+\frac{2}{3} A^{(3 / 2)} .
\end{aligned}
$$

The relation for the cross section of $1 \pi$ photoproduction is given by

$$
\begin{aligned}
& \sigma^{1 \pi}=2 \pi \frac{|\mathbf{q}|}{|\mathbf{k}|} \sum_{l=0}^{\infty}(l+1)^{2}\left[(l+2)\left(\left|E_{l+}\right|^{2}+\left|M_{(l+1)-}\right|^{2}\right)+l\left(\left|M_{l+}\right|^{2}+\left|E_{(l+1)-}\right|^{2}\right)\right] \\
& \Delta \sigma^{1 \pi}=2 \pi \frac{|\mathbf{q}|}{|\mathbf{k}|} \sum_{l=0}^{\infty}(l+1)^{2}(-1)^{l}\left[(l+2)\left(\left|E_{l+}\right|^{2}-\left|M_{(l+1)-}\right|^{2}\right)+l\left(\left|M_{l+}\right|^{2}-\left|E_{(l+1)-}\right|^{2}\right)\right] \\
& \Delta \sigma^{1 \pi}=\sigma^{1 \pi}(E 1, M 2, \cdots)-\sigma^{1 \pi}(M 1, E 2, \cdots)
\end{aligned}
$$

The peak cross section $I_{r}$ introduced in (17) is given by

$$
I_{r}=2 \pi \frac{1}{k_{r}^{2}} \frac{2 J+1}{2 J_{0}+1} \frac{\Gamma_{\gamma}}{\Gamma},
$$


where $J$ and $J_{0}$ are the spins of the excited state and the ground state, respectively, $\Gamma_{\gamma}$ the photon width and $\Gamma$ the total width of the resonance. The photon width $\Gamma_{\gamma}$ may be expressed through the resonance couplings $A_{1 / 2}$ and $A_{3 / 2}$ by the relation [16]

$$
\Gamma_{\gamma}=\frac{k_{r}^{2}}{\pi} \frac{2 M_{N}}{(2 J+1) M_{R}}\left[\left|A_{1 / 2}\right|^{2}+\left|A_{3 / 2}\right|^{2}\right]
$$

where $M_{N}$ and $M_{R}$ are the nucleon and resonant masses. Combining (35) and (36) we arrive at 3

$$
I_{r}=\frac{2 M_{N}}{M_{R} \Gamma}\left[\left|A_{1 / 2}\right|^{2}+\left|A_{3 / 2}\right|^{2}\right] .
$$

Using (37) the quantity $I_{r}$ can be calculated from the resonance couplings $A_{1 / 2}$ and $A_{3 / 2}$ given by the PDG [16], by GWSES [17] and Mainz [19]. The results obtained for the electromagnetic polarizabilities obtained from the data given in [19] are given in lines $3-7$ of Table 2 .

Table 2: Partial contributions to the electromagnetic polarizabilities. The resonant contributions in lines 3-7 are obtained from the analysis of Drechsel et al. [19]. The $t$-channel parts in line 8 are the predictions based on the $\sigma$-meson pole representation (see section 2). The predicted contribution due to the $E_{0+}$ amplitude in line 9 is based on the analyses given in [17-19]. Line 10 contains the differences between the numbers in line 2 and the sums of numbers given in lines $3-9$. The experimental data are normalized to $(\alpha+\beta)_{p}=13.9 \pm 0.3$ and $(\alpha+\beta)_{n}=15.2 \pm 0.5$

\begin{tabular}{|c|c|c|c|c|c|}
\hline 1 & & $\alpha_{p}$ & $\beta_{p}$ & $\alpha_{n}$ & $\beta_{n}$ \\
\hline 2 & experiment & $12.0 \pm 0.6$ & $1.9 \mp 0.6$ & $12.5 \pm 1.7$ & $2.7 \mp 1.8$ \\
\hline 3 & $P_{33}(1232) M_{1+}^{(3 / 2)}, E_{1+}^{(3 / 2)}$ & -1.1 & +8.3 & -1.1 & +8.3 \\
\hline 4 & $P_{11}(1440){ }_{p, n} M_{1-}^{(1 / 2)}$ & -0.0 & +0.2 & -0.0 & +0.1 \\
\hline 5 & $D_{13}(1520){ }_{p, n} E_{2-}^{(1 / 2)},{ }_{p, n} M_{2-}^{(1 / 2)}$ & +0.6 & -0.2 & +0.5 & -0.1 \\
\hline 6 & $S_{11}(1535){ }_{p, n} E_{0+}^{(1 / 2)}$ & +0.1 & -0.0 & +0.1 & -0.0 \\
\hline 7 & $F_{15}(1680){ }_{p, n} E_{3-}^{(1 / 2)},{ }_{p, n} M_{3-}^{(1 / 2)}$ & -0.1 & +0.3 & -0.0 & +0.0 \\
\hline 8 & $t$-channel & +7.6 & -7.6 & +7.6 & -7.6 \\
\hline 9 & $E_{0+}($ empirical $)$ & +3.2 & -0.3 & +4.1 & -0.4 \\
\hline 10 & background & +1.7 & +1.2 & +1.3 & +2.4 \\
\hline
\end{tabular}
(see $[6])$.

The main contributions to the nonresonant parts of the electromagnetic polarizabilities are expected from the $E_{0+}$ amplitude which has to be taken from analyses of meson photoproduction data. Multipole analyses of pion photoproduction based on fixed- $t$ dispersion relations and unitarity are given by Hanstein et al. [18] in a convenient form. Cross sections separated into resonant and nonresonant parts are provided for the reactions $\gamma p \rightarrow \pi^{+} n$ and $\gamma n \rightarrow \pi^{-} p$ up to energies of $500 \mathrm{MeV}$ and extrapolations of the nonresonant parts are straightforward using the data contained in [19] and [17]. In principle there is a problem in disentangling resonant and nonresonant contributions because of interference effects. The interference of the amplitudes ${ }_{p, n} E_{0+}^{(1 / 2)}$ with the $S_{11}(1535)$ and $S_{11}(1650)$ resonances, however, does not lead to problems in determining the nonresonant $E_{0+}$ contributions because of the smallness of the resonant parts. The results for the electromagnetic polarizabilities obtained from these empirical $E_{0+}$ data are contained in line 9 of Table 2 .

\footnotetext{
${ }^{3}$ It should be noted that the quantity $I_{r}$ of (37) contains the branching correction $\Gamma / \Gamma_{\pi}$ as required.
} 
Up to this point the electromagnetic polarizabilities find an explanation in the numbers given in lines $3-9$ of Table 2, with the exception of the small contributions given in line 10 which deserve a further investigation. These non- $E_{0+}$ parts of the nonresonant contributions are partly due to the $M_{1-}^{(3 / 2)},{ }_{p, n} M_{1+}^{(1 / 2)}$ and ${ }_{p, n} E_{1+}^{(1 / 2)}$ amplitudes which interfere with the corresponding resonant amplitudes ${ }_{p, n} M_{1-}^{(1 / 2)}\left(P_{11}(1440)\right), M_{1+}^{(3 / 2)}\left(P_{33}(1232)\right)$ and $E_{1+}^{(3 / 2)}\left(P_{33}(1232)\right)$, respectively [19]. Only the nonresonant parts of the $M_{1-}$ and $M_{1+}$ amplitudes are expected to be to some extent important in comparison with dominant $E_{0+}$ amplitude. Therefore we restrict the present discussion to the $M_{1-}$ and $M_{1+}$ amplitudes. Using the data given in [19] we arrive at the estimates

$\alpha_{p}^{\text {nonres. }}\left(M_{1-}\right)=-0.0, \beta_{p}^{\text {nonres. }}\left(M_{1-}\right)=+0.2, \alpha_{n}^{\text {nonres. }}\left(M_{1-}\right)=-0.1, \beta_{p}^{\text {nonres. }}\left(M_{1-}\right)=+0.4$, $\alpha_{p}^{\text {nonres. }}\left(M_{1+}\right)=-0.0, \beta_{p}^{\text {nonres. }}\left(M_{1+}\right)=+0.3, \alpha_{n}^{\text {nonres. }}\left(M_{1+}\right)=-0.1, \beta_{p}^{\text {nonres. }}\left(M_{1+}\right)=+0.6$.

The conclusion we have to draw from this is that it is not possible to relate the numbers given in line 10 of Table 2 to known photoproduction processes, unless the two-pion channels are taken into account (see e.g. [23]). The $\pi \pi N$ final states can be characterized either as quasi twobody states such as $\pi \Delta$ and $\rho N$, or as a $\pi \pi N$ component in which both pions are in $S$ waves. Furthermore, in the Regge regime above $\approx 2000 \mathrm{MeV}$ also $f_{2}(1270), a_{2}(1320)$ and Pomeron $t$-channel exchanges play a role. The $\pi \Delta$ contribution has been analyzed in terms of a $\Delta$ KrollRuderman term and a $\Delta$ pion-pole term [24]. Using data from this analysis [24] we arrive at $\left(\alpha_{p, n}+\beta_{p, n}\right) \approx 1.0$ for this partial $\pi \pi$ channel. The nonresonant cross section above $\approx 2000$ $\mathrm{MeV}$ makes a contribution of about $\left(\alpha_{p, n}+\beta_{p, n}\right) \approx 0.7$.

\section{Discussion}

\subsection{Discussion of the $s$-channel contribution}

For a long time there have been attempts to understand the electromagnetic polarizabilities predominantly in terms of properties of the "pion cloud" of the nucleon. Among these attempts CHPT in its original relativistic form [25] is among the most prominent ones. It has been shown by L'vov [26] that the results obtained for the electromagnetic polarizabilities through the evaluation of chiral loops [25] can be reproduced via dispersion theory when the Born approximation of the electric-dipole CGLN amplitude $E_{0+}$ is taken into account. The results obtained in this way are shown in lines 2 and 3 of Table 3 .

Table 3: Predictions for the "meson cloud" contribution to the electromagnetic polarizabilities in different approaches.

\begin{tabular}{ll|cc|cc|l}
\hline 1 & method & $\alpha_{p}$ & $\beta_{p}$ & $\alpha_{n}$ & $\beta_{n}$ & reference \\
\hline 2 & CHPT & +7.4 & -2.0 & +10.1 & -1.2 & Bernard [25] \\
3 & pion $^{a)}$ Born & +7.3 & -1.8 & +9.8 & -0.9 & L'vov [26] \\
4 & E0+ Born & +7.5 & -1.4 & +9.9 & -1.8 & present \\
\hline
\end{tabular}

a) The use of fixed- $t$ dispersion theory requires the consideration of interference terms of the $E_{0+}$ amplitude with other amplitudes.

It is of interest to use also the present approach based on forward and backward dispersion relations for studies of this type. For this purpose use may be made of the Born approximation 
(see [22] p. 286, [27] p. 35) given in the form

$$
\begin{aligned}
& E_{0+}^{\text {Born }}\left(\gamma N \rightarrow \pi^{ \pm} N\right)= \pm \sqrt{2}\left(E_{0+}^{(-) \text {Born }} \pm E_{0+}^{(0) \text { Born }}\right), \\
& E_{0+}^{(-) \text {Born }}=\frac{e f}{4 \pi m_{\pi}}\left[1-\frac{1}{2}\left(1+\frac{1-v^{2}}{2 v} \ln \left(\frac{1-v}{1+v}\right)\right)\right],
\end{aligned}
$$

with $v=|\mathbf{q}| / \sqrt{\mathbf{q}^{2}+m_{\pi}^{2}}$ being the velocity of the pion in the c.m. system. The expression given in (39) corresponds to the static approximation discussed in detail in [22,27]. Because of the relation

$$
\frac{\sigma_{E_{0+}}\left(\gamma n \rightarrow \pi^{-} p\right)}{\sigma_{E_{0+}}\left(\gamma p \rightarrow \pi^{+} n\right)}=\left(1+\frac{m_{\pi}}{m}\right)^{2} \simeq 1.3
$$

(see $[22]$ p. 276$)$ the recoil terms $E_{0+}^{(0) \text { Born }}$ may be replaced by multiplying $E_{0+}^{(-) \text {Born }}$ with $(1+$ $\left.\frac{m_{\pi}}{m}\right)^{-1 / 2}$ and $\left(1+\frac{m_{\pi}}{m}\right)^{+1 / 2}$ in order to get the results for the proton and neutron, respectively. The relation given in (40) is well justified at threshold but its approximate validity extends to higher energies $[18,19,27]$. The pseudovector coupling constant $f$ in (39) is given by $f=g_{\pi N N}\left(m_{\pi} / 2 m\right)$ with $g_{\pi N N}=13.169 \pm 0.057$. There is a remarkable agreement between the numbers given in Table 3 but these numbers are larger by a factor $\sim 2.4$ than the corresponding numbers in line 9 of Table 2. Two reasons for the deviation of the empirical $E_{0+}$ amplitude from the Born approximation have been discussed in [19]. The first reason is that the pseudovector (PV) coupling is not valid at high photon energies but has to be replaced by some average of the PV and pseudoscalar the (PS) coupling. The second reason are $\rho$ and $\omega$ meson $t$-channel exchanges which are not taken into account in the Born approximation.

In Table 2 (see also Table 1) we see that the different resonant contributions to the electric polarizabilities cancel each other, so that the electric polarizabilities are mainly due to the $t$-channel part $\alpha_{p, n}^{t}(\sim 60 \%)$ given in line 8 and a smaller nonresonant part $\alpha\left(E_{0+}\right)(\sim 30 \%)$ given in line 9 . For the magnetic polarizabilities there is an almost complete cancellation of the $P_{11}(1440), D_{13}(1520)$ and $F_{15}(1680)$ contributions, so that the main remaining contributions are due to the $P_{33}(1232)$ resonance, canceled to a large extent by the $t$-channel contribution $\beta^{t}$. The nonresonant background given in line 10 of Table 2 amounts to about $10 \%$ of the experimental electric polarizabilities and to about $70 \%$ of the experimental magnetic polarizabilities. This means that precise predictions of these contributions are highly desirable, especially for the magnetic polarizabilities. Unfortunately, the non- $E_{0+}$ parts of the nonresonant photoabsorption cross sections are dominated by two-pion channels where the information on the multipole content is scarce.

\subsection{Discussion of the $t$-channel contribution}

In [1] it has been shown that there are two independent, but apparently equivalent and complementary options to calculate the scalar-isoscalar $t$-channel contribution to the electromagnetic polarizabilities of the nucleon.

Option 1 makes use of the properties of the $\sigma$-meson as predicted by the quark-level NJL model and in this respect is of course model dependent. The quark-level NJL model predicts a definite $\sigma$-mesons mass, viz. $m_{\sigma}=666 \mathrm{MeV}$, through a parameter-free relation of $m_{\sigma}$ to the pion decay constant $f_{\pi}$. The result $(\alpha-\beta)^{t}=15.2$ is in an excellent agreement with the experimental result. The agreement between a prediction and an experimental result cannot be used as an argument for the validity of the prediction without further support. This support is provided by dispersion theory applied to the measured properties of the $\sigma$ meson as showing up in particle reactions with two pions in the intermediate state (Option 2). 
Option 2 first takes into consideration that the $\sigma$ meson has been observed in many data analyses [16] as a pole on the second sheet of the isoscalar $S$ wave of $\pi \pi$ scattering. This pole describes part of the resonant structure of the $\sigma$ meson without being a complete description. This latter property of the pole follows from the fact that the $90^{\circ}$ crossing of the scalar-isoscalar phase $\delta_{0}^{0}(s)$ is located at much higher energies than predicted by the structure of the pole. The analyses of Colangelo et al. [28] and Caprini et al. [29] led to

$$
\begin{aligned}
& \sqrt{s}(\text { pole })=(470 \pm 30)-i(295 \pm 20) \mathrm{MeV} \quad \sqrt{s}\left(\delta_{S}=90^{\circ}\right)=(844 \pm 13) \mathrm{MeV}[28] \\
& M_{\sigma}=441_{-8}^{+16} \mathrm{MeV}, \quad \Gamma_{\sigma}=544_{-25}^{+18} \mathrm{MeV}[29] .
\end{aligned}
$$

The numbers contained in (41) and (42) are extremely valuable in characterizing the properties of the $\sigma$ meson as a real particle but they can only qualitatively be compared with the mass $m_{\sigma}=666 \mathrm{MeV}$ of the virtual $\sigma$ meson, because in the latter case there is no open decay channel. This means that there is no contradiction between the existence of the broad mass distribution for the real $\sigma$ meson and a precisely determined mass of the virtual $\sigma$ meson. Furthermore, the numbers contained in (41) and (42) are of no direct relevance for the prediction of $(\alpha-\beta)^{t}$. First of all it certainly would lead only to a qualitative estimate for $(\alpha-\beta)^{t}$ if the parameters of the $\sigma$-meson pole in (411) and (42) would be used instead of $m_{\sigma}=666 \mathrm{MeV}$. Furthermore, such an insufficient attempt is not necessary because the BEFT [4] sum rule provides a precise relation between $(\alpha-\beta)^{t}$ and the properties of the real $\sigma$ meson. In the BEFT sum rule the imaginary part of the $t$-channel Compton scattering amplitude is given by an unitarity relation where the two reaction $\gamma \gamma \rightarrow \sigma \rightarrow \pi \pi$ and $N \bar{N} \rightarrow \sigma \rightarrow \pi \pi$ are exploited. In these reactions the resonant structure of the $\sigma$ meson enters via the experimentally determined scalar-isoscalar phase $\delta_{0}^{0}(s)$ which is considerably different from the corresponding quantity predicted by the poles shown in (41) and (42). The real part of the $t$-channel Compton scattering amplitude is obtained via a dispersion relation. The present status of the evaluation of the BEFT sum rule $(\alpha-\beta)_{n p}^{t}=15.3 \pm 1.3$ is in good agreement with the experimental result as well as the prediction based on the quark-level NJL model.

\section{Conclusion}

The good agreement of the result based on the BEFT sum rule with the experimental result as well as the prediction based on the quark level NJL model may be understood as a strong argument that the two predictions of $(\alpha-\beta)^{t}$ are equivalent. This implies that in addition to the poles in (41) and (42) also the mass $m_{\sigma}=666 \mathrm{MeV}$ of the virtual $\sigma$ meson is an experimentally verified property of the $\sigma$ meson.

\section{Acknowledgment}

The author is indebted to Deutsche Forschungsgemeinschaft for the support of this work through the projects SCHU222 and 436RUS113/510. He thanks M.I. Levchuk, A.I. L'vov and A.I. Milstein for a long term cooperation which contributed to the motivation for the present investigation. 


\section{References}

[1] M. Schumacher, Eur. Phys. J. A 30, 413 (2006); DOI 10.1140/epja/i2006-10103-0 hep-ph/0609040.

[2] A.C. Hearn, E. Leader, Phys. Rev. 126, 789 (1962); R. Köberle, Phys. Rev. 166, 1558 (1968).

[3] E.E. Low, Phys. Rev. 120, 582 (1960) (and reference therein); M. Jacob, J. Mathews, Phys. Rev. 117, 854 (1960).

[4] J. Bernabeu, T.E.O. Ericson, C. Ferro Fontan, Phys. Lett. 49 B, 381 (1974); J. Bernabeu, B. Tarrach, Phys. Lett 69 B, 484 (1977).

[5] I. Guiasu, E.E. Radescu, Phys. Rev. D 14, 1335 (1976); Phys. Lett. 62 B, 193 (1976).

[6] M. Schumacher, Prog. Part. Nucl. Phys. 55, 567 (2005) hep-ph/0501167.

[7] B.R. Holstein, A.M. Nathan, Phys. Rev. D 49, 6101 (1994).

[8] M.I. Levchuk, A.I. L'vov, A.I. Milstein, M. Schumacher, Proceedings of the Workshop NSTAR2005, 12-15 October 2005, Tallahassee, Florida, edited by S. Capstick, V. Crede, P. Eugenio (World Scientific 2006) 389 hep-ph/0511193].

[9] D. Drechsel et al., Phys. Rep. 378, 99 (2003); Phys. Rev. C 61, 015204 (1999).

[10] T. Hatsuda, T. Kunihiro, Phys. Rep. 247, 221 (1994).

[11] R. Delbourgo, M. Scadron, Mod. Phys. Lett. A 10, 251 (1995) hep-ph/9910242]; Int. J. Mod. Phys. A 13, 657 (1998) hep-ph/9807504.

[12] A.I. L'vov, V.A. Petrun'kin, M. Schumacher, Phys. Rev. C 55, 359 (1997).

[13] G.F. Chew, M.L. Goldberger, F.E. Low, Y. Nambu, Phys. Rev. 106, 1345 (1957).

[14] A.M. Baldin, Nucl. Phys. 18, 310 (1960); L.I. Lapidus, Zh. Eksp. Teor. Fiz. 43, 1358 (1962) [Sov. Phys. JETP 16, 964 (1963)].

[15] T.A. Armstrong et al., Phys. Rev. D 5, 1640 (1972); Nucl. Phys. B 41, 445 (1972).

[16] W.-M. Yao et al., (Particle Data Group) J. Phys. G 33, 1 (2006) [URL: http://pdg.lbl.gov].

[17] R.A. Arndt, et al. Phys. Rev. C 66, 055213 (2002).

[18] O. Hanstein, D. Drechsel, L. Tiator, Nucl. Phys. A 632, 561 (1998).

[19] D. Drechsel, O. Hanstein, S.S. Kamalov, L. Tiator, Nucl. Phys. A 645, 145 (1999).

[20] R.L. Walker, Phys. Rev. 182, 1729 (1969).

[21] K.M. Watson, Phys. Rev. 95, 228 (1954).

[22] T. Ericson, W. Weise, Pions and Nuclei, International Series of Monographs on Physics 74, Oxford Science Publications (1988).

[23] D. Drechsel, L. Tiator, J. Phys. G: Nucl. Part. Phys. 18, 449 (1992).

[24] J.A. Gómez Tejedor, E. Oset, Nucl. Phys. A 571, 667 (1994); 600, 413 (1996). 
[25] V. Bernard, N. Kaiser, U.-G. Meissner, Phys. Rev. Lett. 67, 1515 (1991); Nucl. Phys. B 373, 346 (1992).

[26] A.I. L'vov, Phys. Lett. B 304, 29 (1993).

[27] A. Donnachie, in: High Energy Physics, Edited by E.H.S. Burhop V, 1 Academic Press (1972)

[28] G. Colangelo, J. Gasser, H. Leutwyler, Nucl. Phys. B 603, 125 (2001).

[29] I. Caprini, G. Colangelo, H. Leutwyler, Phys. Rev. Lett. 96, 132001 (2006). 